\title{
Structures for $M$-Channel Perfect-Reconstruction FIR QMF Banks Which Yield Linear-Phase Analysis Filters
}

\author{
TRUONG Q. NGUYEN, MEMBER, IEEE, AND P. P. VAIDYANATHAN, SENIOR MEMBER, IEEe
}

\begin{abstract}
In this paper, we develop structures for FIR perfect-reconstruction QMF banks which cover a subclass of systems that yield linear-phase analysis filters for arbitrary $M$. The parameters of these structures can be optimized in order to design analysis filters with minimum stopband energy which at the same time have linear-phase and satisfy the perfect-reconstruction property. If there are $M$ subbands, then depending upon whether the coefficients $h_{k}(n)$ of each analysis filter is symmetric or antisymmetric, several combinations of filter banks are possible. Some of these permit perfect-reconstruction and some do not. For a given $M$, we develop a formula for the number of combinations for a subclass of linear-phase perfect-reconstruction structures. As an example, we elaborate on a perfect-reconstruction linear-phase lattice structure for three channels and develop a lattice structure for this case. The lattice structure is such that, regardless of the parameter values, the QMF bank enjoys perfect-reconstruction property while at the same time the analysis filters have linear phase. These parameters can therefore be optimized to obtain analysis filters with good magnitude response, without losing the above two features. A design example, based on optimization of the parameters in the lattice structure, is presented, along with tables of impulse response coefficients.
\end{abstract}

\section{INTRODUCTION}

$\mathrm{Q}$ UADRATURE mirror filters (in short, QMF) are used in many speech and communications applications [1]-[3]. Throughout this paper, we consider the maximally decimated $M$-channel quadrature mirror filter (QMF) bank shown in Fig. 1. The analysis filters $H_{k}(z)$ split the transmitted signal $x(n)$ into $M$ subband signals which in turn are decimated by $M$ and encoded prior to transmission. At the receiving end, the $M$ subband signals are decoded, interpolated, and recombined using the synthesis filters $F_{k}(z)$. Ignoring the nonideal channel characteristic and the nonlinear encoding/decoding error, the signal $\hat{x}(n)$ suffers from four errors [5], namely, aliasing due to decimation, imaging due to interpolation [2], amplitude distortion, and phase distortion. The last two errors are due to the nonideal nature of the analysis filters. In this paper, we deal with FIR QMF banks in which all four above distortions are eliminated (aliasing effects

Manuscript received July 29, 1988; revised May 15, 1989. This work was supported in part by the National Science Foundation under Grants DCI 8552579 and MIP 8604456. T. Q. Nguyen was an Aerojet Fellow during the first haif of the period when this work was performed.

The authors are with the Department of Electrical Engineering, California Institute of Technology, Pasadena, CA 91125.

IEEE Log Number 8933424.

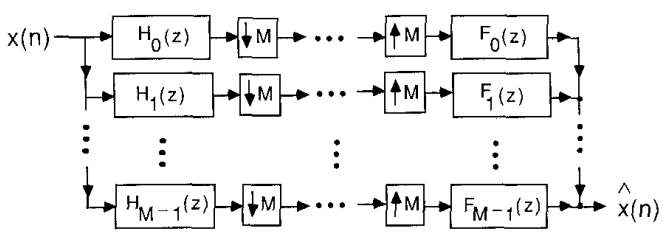

Fig. 1. The $M$-channel maximally decimated parallel QMF bank.

being cancelled by imaging effects), so that $\hat{x}(n)$ is a delayed version of $x(n)$, i.e., $\hat{x}(n)=c x\left(n-n_{0}\right), c \neq 0$. Such structures are called perfect-reconstruction (abbreviated as PR) structures, and the QMF bank of Fig. 1 is then said to be a PR system.

Each analysis filter $H_{k}(z)$ and synthesis filter $F_{k}(z)$ in Fig. 1 can be written in the form $H_{k}(z)=\Sigma_{l=0}^{M-1}$ $z^{-l} E_{k l}\left(z^{M}\right)$ and $F_{k}(z)=\sum_{l=0}^{M-1} z^{-(M-1-l)} R_{l k}\left(z^{M}\right)$, respectively. The quantities $E_{k l}(z)$ and $R_{l k}(z), 0 \leq l \leq M$ - 1 are the $M$ polyphase components [2], [13], [14] of $H_{k}(z)$ and $F_{k}(z)$, respectively. Defining the two $M \times M$ matrices $\boldsymbol{E}(z)=\left[E_{k l}(z)\right]$ and $\boldsymbol{R}(z)=\left[\boldsymbol{R}_{l k}(z)\right]$, which are called the polyphase component matrices for the analysis bank and synthesis bank, respectively, the QMF bank of Fig. 1 can be redrawn as in Fig. 2. Using standard identities in multirate signal processing [2], [13], Fig. 2 can be further reduced to Fig. 3. Therefore, we can obtain a perfect-reconstruction system if we choose [12] $R(z)=$ $\boldsymbol{E}^{-1}(z)$. If the analysis filters are FIR, the choice $\boldsymbol{R}(z)=$ $\boldsymbol{E}^{-1}(z)$ gives rise to FIR synthesis filters as well, provided that det $\boldsymbol{E}(z)=b z^{-r}$, where $b$ and $r$ are a nonzero constant and a nonnegative integer, respectively. In this paper, the term "perfect-reconstruction" is taken to be synonymous to the condition $\operatorname{det} \boldsymbol{E}(z)=b z^{-r}$ (even though this is not a necessary condition with IIR perfectreconstruction systems).

The theory of perfect reconstruction when $M$ is a power of 2 is well known [6], [15]. The design method in [6] is based on spectral factorization of an FIR halfband filter. Some methods of perfect reconstruction for arbitrary number of channels have been reported recently [9], [12]. The method described in [12] constrains the polyphase transfer matrix $\boldsymbol{E}(z)$ to be FIR and lossless (i.e., $\boldsymbol{E}\left(e^{j \omega}\right)$ to be unitary for all $\omega)$. The coefficients here are assumed to be real. Under this condition, if the matrix $\boldsymbol{R}(z)$ is cho- 


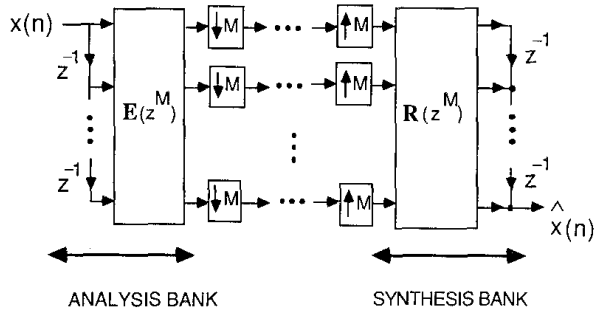

Fig. 2. Polyphase implementation of Fig. 1.

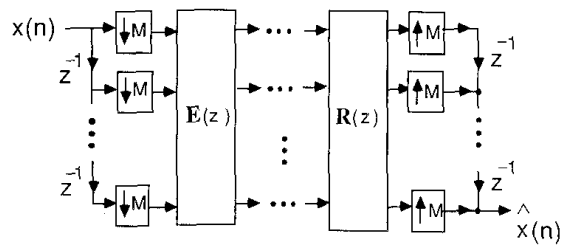

Fig. 3. An equivalent structure of Fig. 2.

sen as $\boldsymbol{R}(z)=\boldsymbol{E}^{T}\left(z^{-1}\right)$, then the system of Fig. 1 is forced to be a PR system. In [10] and [16], a procedure for design of two-channel perfect-reconstruction systems with linear-phase FIR filters is given, based on judicious factorization of a linear-phase FIR halfband filter. The number of possible spectral factors, hence, grows exponentially with respect to the degree of the filters; besides, the resulting filters are not guaranteed to be optimal. In a recent paper [17], the authors discussed several properties of $M$-channel linear-phase perfect-reconstruction systems which yield equal length analysis filters. They also studied lattice structures for the two-channel linear-phase perfect-reconstruction bank in [18]; the analysis filters $H_{k}(z)$ of these structures obey several conditions as follows:

1) the sum of their lengths is a multiple of 4 , and

2) the filters have odd degrees and opposite symmetry or they have even degrees and same symmetry.

In a similar way, one of our goals in this paper is to obtain corresponding results for the $M$-channel linearphase perfect-reconstruction FIR QMF bank. ${ }^{1}$ This is done in Section II. As in the two-channel case, these results should reflect into the sum of the lengths of the analysis filters. Moreover, we also give a count of the number of distinct combinations which could yield linear-phase FIR analysis filters for a subclass of PR structures. (We will state which subclass we consider here in Section II.) Our restriction to this subclass is primarily motivated by analytic tractability.

We first derive the necessary form of $E(z)$ such that $H_{k}(z), 0 \leq k \leq M-1$ are linear phase FIR analysis filters in Section II. Having limited our study to a subclass of these PR structures, the conditions on the lengths and the symmetries of $H_{k}(z)$ are derived by imposing the perfect-reconstruction condition on the above $\boldsymbol{E}(z)$. We will

Independent work in this direction has also been reported in [22]. also give the exact number of linear-phase perfect-reconstruction structures for this subclass. In Section III, we demonstrate the practical feasibility of these structures by concentrating on obtaining a lattice structure for the 3-channel case. Once this linear-phase perfect-reconstruction lattice structure for 3-channel QMF bank is obtained, the pairwise-symmetric property [19] is incorporated on it to speed up the convergence time in the design process. A design example is also included in Section III.

Notations Used in the Paper: We consider only realcoefficient linear-phase FIR filters in this paper. Boldfaced italic letters indicate vectors and matrices, whereas superscript $T$ denotes transposition. If the impulse response $h(n)$ of $H(z)$ is symmetric, we say " $H(z)$ is symmetric," and so on. The center of a linear phase FIR transfer function is defined to be the center of symmetry or antisymmetry of $h(n)$. Clearly, the center of $H(z)$ could be either an integer or an odd multiple of $(1 / 2)$. The tilde accent - on a function $F(z)$ is defined such that $\tilde{F}(z)=F^{T}\left(z^{-1}\right)$. The mirror image of $H(z)$, denoted by $\hat{H}(z)$ is defined as $\hat{H}(z) \triangleq z^{-(N-1)} H\left(z^{-1}\right)$. Here $h(N-$ $1)$ is the highest nonzero coefficient of $H(z)$ and $N-1$ is called the degree of $H(z)$. For brevity, "linear phase", and "perfect reconstruction" are abbreviated as LP and $P R$, respectively.

\section{II. $M$-ChANNEL LP PR FIR QMF BANKS}

Let $H_{k}(z), 0 \leq k \leq M-1$ be the $M$ (causal) linearphase analysis filters with center $c_{k}$. We define the degree of $H_{k}(z)$ as $N_{k}-1 \triangleq 2 c_{k}$. For a given $M$, we can uniquely write $N_{k}-1$ as $m_{k} M+i_{k}$ where $0 \leq i_{k} \leq M-1$ and $m_{k}$ are integers. This representation will be used subsequently. In terms of the polyphase components $E_{k j}(z)$, the filter $H_{k}(z)$ is represented as [12]

$$
H_{k}(z)=\sum_{j=0}^{M-1} z^{-j} E_{k j}\left(z^{M}\right) .
$$

We shall define the center of $E_{k j}(z)$ as $c_{k j}$ where

$$
2 c_{k j} \triangleq \begin{cases}m_{k}, & j \leq i_{k} ; \\ m_{k}-1, & j>i_{k} .\end{cases}
$$

First, let us find the relations among the polyphase component $E_{k j}(z)$ which yields linear-phase analysis filters. Once these relations are known, we can further study the perfect-reconstruction aspect of these structures.

The impulse response of a linear-phase filter $H_{k}(z)$ could be either a symmetric or an antisymmetric sequence, i.e.,

$$
\tilde{H}_{k}(z)=z^{N_{k}-1} J_{k} H_{k}(z)
$$

where

$$
J_{k}=\left\{\begin{aligned}
1, & H_{k}(z) \text { is symmetric } \\
-1, & H_{k}(z) \text { is antisymmetric. }
\end{aligned}\right.
$$


Using (1) in (3) and noting that $N_{k}-1=m_{k} M+i_{k}$, we obtain

$$
\begin{aligned}
\sum_{j=0}^{M-1} z^{j} \tilde{E}_{k j}\left(z^{M}\right) & \\
= & z^{\left(m_{k} M+i_{k}\right)} J_{k} \sum_{j=0}^{M-1} z^{-j} E_{k j}\left(z^{M}\right) \\
= & z^{\left(m_{k} M+i_{k}\right)} J_{k} \sum_{j=0}^{i_{k}} z^{-j} E_{k j}\left(z^{M}\right) \\
& +z^{\left(m_{k} M+i_{k}\right)} J_{k} \sum_{j=i_{k}+1}^{M-1} z^{-j} E_{k j}\left(z^{M}\right) \\
= & J_{k} \sum_{j=0}^{i_{k}} z^{(i k-j)}\left[z^{m_{k} M} E_{k j}\left(z^{M}\right)\right] \\
& +J_{k} \sum_{j=i_{k}+1}^{M-1} z^{\left(M+i_{k}-j\right)}\left[z^{\left(m_{k}-1\right) M} E_{k j}\left(z^{M}\right)\right] .
\end{aligned}
$$

Making changes of variables, the above equation becomes

$$
\begin{aligned}
\sum_{j=0}^{M-1} z^{j} & \tilde{E}_{k j}\left(z^{M}\right) \\
= & J_{k} \sum_{j=0}^{i_{k}} z^{j}\left[z^{m_{k} M} E_{k, i_{k}-j}\left(z^{M}\right)\right] \\
& \quad+J_{k} \sum_{j=i_{k}+1}^{M-1} z^{j}\left[z^{\left(m_{k}-1\right) M} E_{k, M+i_{k}-j}\left(z^{M}\right)\right] .
\end{aligned}
$$

Comparing like powers of both sides of (6), we have the following necessary form of $\boldsymbol{E}(z)$ which yields linearphase analysis filters. Thus,

$$
\tilde{E}_{k j}(z)=z^{m_{k}} J_{k} \times \begin{cases}E_{k,(i k-j)}(z), & j \leq i_{k} ; \\ z^{-1} E_{k,\left(M+i_{k}-j\right)}(z), & j>i_{k} .\end{cases}
$$

The above relation has been known before in a slightly different notation [11]. Let us now concentrate on a subclass of systems that satisfy (7), namely, the class where all $i_{k}$ are equal, i.e., $i_{0}=i_{1}=\cdots=i_{M-1}=I$. From now on, we only consider this class. The restriction of our discussion to this class is motivated primarily by the tractability of the possible combinations of PR systems belonging to this subclass.

Recalling that $N_{k}=m_{k} M+i_{k}+1$, we see that for this particular subclass, the sum of the lengths of the analysis filters is a multiple of $M . E(z)$ in (7) becomes

$$
\tilde{E}_{k j}(z)=z^{m_{k}} J_{k} \times \begin{cases}E_{k,(I-j)}(z), & j \leq I ; \\ z^{-1} E_{k,(M+I-j)}(z), & j>I .\end{cases}
$$

The above form of $\boldsymbol{E}(z)$ yields a linear-phase QMF structure. It does not, however, guarantee perfect reconstruction yet. To achieve this additional goal, namely, perfect reconstruction, let us study $\boldsymbol{E}(z)$ carefully. It can be ver- ified that (8) is equivalent to

$$
\tilde{E}(z)=\Lambda_{1}(z) \boldsymbol{P} E^{T}(z) \mathbf{\Lambda}_{2}(z)
$$

where $\boldsymbol{\Lambda}_{1}(z)$ and $\boldsymbol{\Lambda}_{2}(z)$ are diagonal matrices such that

$$
\left(\boldsymbol{\Lambda}_{1}\right)_{j j}=\left\{\begin{array}{cl}
1, & j \leq I ; \\
z^{-1}, & j>I,
\end{array} \quad \text { and }\left(\boldsymbol{\Lambda}_{2}\right)_{j j}=z^{m_{j}} J_{j}\right.
$$

Here, $\boldsymbol{P}$ is a permutation matrix of the form

$$
\boldsymbol{P}=\left(\begin{array}{ll}
\Gamma_{(I+1)} & \mathbf{0} \\
\mathbf{0} & \Gamma_{(M-1-I)}
\end{array}\right)
$$

where

$$
\Gamma_{I}=\left(\begin{array}{cc}
\mathbf{0} & 1 \\
& \therefore
\end{array}\right)_{(I \times I)}
$$

For a PR system, det $\boldsymbol{E}(z)=b z^{-r}$. Taking the determinant of both sides in (9),

$$
\operatorname{det} \tilde{\boldsymbol{E}}(z)=\operatorname{det} \boldsymbol{\Lambda}_{1}(z) \operatorname{det} \boldsymbol{P} \operatorname{det} \boldsymbol{E}^{T}(z) \operatorname{det} \boldsymbol{\Lambda}_{2}(z)
$$

or, equivalently

$$
b z^{r}=b z^{-(M-1-I)} z^{-r} z^{\left(\Sigma_{k=0}^{M-1} m_{k}\right)}\left(\prod_{k=0}^{M-1} J_{k}\right) \operatorname{det} \boldsymbol{P} .
$$

Comparing both sides of (14), we have the two following conditions:

$$
2 r=\left(\sum_{k=0}^{M-1} m_{k}\right)-(M-1-I)
$$

$$
\left(\prod_{k=0}^{M-1} J_{k}\right) \operatorname{det} \boldsymbol{P}=1
$$

The first equation in (15) yields the same condition on the filter lengths as above. Namely, the sum of the lengths of the analysis filters is a multiple of $M$. From (15b), one can obtain the total number of possible combinations of analysis filters which yield LP PR systems.

We have shown in [18] that, in the case of two-channel LP PR FIR QMF banks, there are only two structures in which all $i_{k}$ are the same. Moreover, they are the only structures that yield nontrivial filters. For an arbitrary number of channels, partly due to the two choices that $J_{k}$ can take (namely, $J_{k}$ can be either 1 or -1 ), and partly due to the $M$ choices that $I$ can take (i.e., $0 \leq I \leq M-$ 1 ), the counting of combinations which yield LP PR FIR QMF banks is not simple. For instance, if $M=3$, there are 3 possible combinations which the triplets $J_{0}, J_{1}$, and $J_{2}$ can take. These are the combinations in which either one or two or all three of the $J_{k}$ are 1 . ( The case where 
none of the $J_{k}$ is 1 , i.e., all $J_{k}$ are -1 , cannot be a PR structure. In other words, the DC component of the signal $x(n)$ cannot be reconstructed since all analysis filter $H_{k}(z)$ have zeros at $\omega=0$ !) Moreover, for each of the above triplets $J_{0}, J_{1}$, and $J_{2}$, there are 3 possibilities because $I$ can be either 0 or 1 or 2 . Thus, there are in total $3^{2}$ structures that yield LP analysis filters in our subclass. In general, there are $M^{2}$ LP structures. Which one of these can be both LP and PR?

Let us turn our attention to (15b) which governs the number of combinations of analysis filters that yield LP PR FIR filters. Let

$$
\left\{\begin{array}{c}
\Delta_{S}=\text { number of combinations of } J_{k} \text { such that } \\
\prod_{k=0}^{S-1} J_{k}=1, \\
\Theta_{S}=\text { number of combinations of } J_{k} \text { such that } \\
\prod_{k=0}^{S-1} J_{k}=-1, \\
R_{1}=\text { number of combinations of } I \text { such that } \\
\text { det } P=1,
\end{array}\right.
$$

then the total number of combinations of analysis filters which are LP PR and are obeying (15b) (denoted as Total) is

$$
\begin{aligned}
\text { Total } & =\Delta_{M} R_{1}+\Theta_{M}\left(M-R_{1}\right) \\
& =R_{1}\left(\Delta_{M}-\Theta_{M}\right)+M \Theta_{M} .
\end{aligned}
$$

Let us calculate $\Delta_{S}, \theta_{S}$, and $R_{1}$ separately as follows. Calculating $R_{1}$ : From (11)

$$
\operatorname{det} \boldsymbol{P}=\operatorname{det} \Gamma_{l+1} \operatorname{det} \Gamma_{M-1-I}
$$

where

$$
\operatorname{det} \Gamma_{k}=\left\{\begin{aligned}
1 ; & k=4 r, 4 r+1, \\
-1 ; & k=4 r+2,4 r+3
\end{aligned}\right.
$$

Here, $r$ is a positive integer. By taking $I$ in the range from 0 to $M-1$ and using (19) in (18), one can verify that

$$
R_{1}= \begin{cases}M / 2 ; & M \text { is even } \\ M ; & M=4 r+1, \\ 0 ; & M=4 r+3,\end{cases}
$$

where $r$ is a positive integer.

Calculating $\Delta_{S}$ : We are interested in the number of combinations of $J_{k}$ for which $\Pi_{k=0}^{S-1} J_{k}=1$ where $J_{k}$ can be either 1 or -1 . As mentioned above, the case where all $J_{k}=-1$ is excluded since PR system is not possible for this case.

Even $S$ : Since $S$ is even, and furthermore since $J_{k}$ can be either 1 or -1 , the number of $J_{k}$ that takes the value -1 has to be even. In other words, there will be 0 , or 2 , $\cdots$, or $S$ values of $J_{k}$ that take the value -1 . That gives us, in total, $(S / 2+1)$ combinations. Excluding the case where all $J_{k}$ are -1 , we have a net total of $S / 2$ combinations in which $\Pi_{k=0}^{S-1} J_{k}=1$.

Odd S: Similar to the above even case, the number of $J_{k}$ that takes the value -1 in this case has to be even. Thus, there will be 0 , or $2, \cdots$, or $(S-1)$ values of $J_{k}$ that takes the value -1 , and thus, $\Delta_{S}=(S+1) / 2$. Combining both cases, $\Delta_{S}$ is

$$
\Delta_{S}= \begin{cases}S / 2 ; & \text { even } S, \\ (S+1) / 2 ; & \text { odd } S .\end{cases}
$$

Calculating $\Theta_{S}$ : Use the same counting argument as in the $\Delta_{S}$ case, where the only difference is that the number of $J_{k}$ taking the values -1 is odd. Thus

$$
\Theta_{S}= \begin{cases}S / 2 ; & \text { even } S, \\ (S-1) / 2 ; & \text { odd } S .\end{cases}
$$

In summary, the total number of LP PR FIR QMF structures is

$$
\text { Total }=R_{1}\left(\Delta_{M}-\theta_{M}\right)+M \Theta_{M},
$$

where $R_{1}, \Delta_{M}$, and $\Theta_{M}$ are as in (20), (21), and (22). We have calculated Total for several $M$ in Table I. We observe that, for $M=2$, this result agrees with previous work [18]. For this particular subclass, let us summarize all results on the LP PR QMF bank in the following fact.

Fact: For an $M$-channel FIR QMF bank in which all $i_{k}$ are the same, the total number of combinations of LP PR analysis filter banks is given in (23). The polyphase transfer matrix $\boldsymbol{E}(z)$ satisfies (8) or, equivalently, (9). The lengths of the analysis filters $H_{k}(z), N_{k}$ satisfy the condition: $\Sigma_{k=0}^{M-1} N_{k}=M(2 r+M)$, where $r$ is some positive integer and $N_{k}-1=m_{k} M+i_{k}$.

From the above discussions, it is clear that there are several possible combinations of analysis filters which yield LP PR FIR QMF banks. In order to develop a design procedure for such systems, we shall adopt the same strategy as in some of our previous work [12], [15]. This strategy is to construct a lattice structure for the analysis bank such that the properties of interest are structurally enforced. In other words, regardless of the values of the multipliers in the structure, the FIR filter bank would satisfy the LP PR property. If we invent such a structure, we can optimize the multipliers in the structure to obtain analysis filter with good stopband attenuations, without sacrificing the LP PR property. 
TABLE I

Numbi:R of COMbinations of LP PR Systems for the CASE Where All. $i_{k}$ ARE THE SAME

\begin{tabular}{|c|c|c|c|c|c|c|}
\hline$M$ & 2 & 3 & 4 & 5 & 6 & 7 \\
\hline Total & 2 & 3 & 8 & 15 & 18 & 21 \\
\hline
\end{tabular}

Now, because of the existence of several possible combinations of analysis filters giving rise to the LP PR property, it is not possible to find a single general lattice structure that covers all of these cases. Our main purpose in the next section is to demonstrate that it is indeed possible to obtain lattice structures if we restrict the filter bank to be a subclass of all the possible combinations. To be specific, if we set $I=M-1$ in the above discussions, it turns out to be easy to obtain such lattice structures.

The purpose of the next section is to demonstrate this with the help of a three-channel QMF bank so that we have $M=3$ and $I=M-1=2$. Even with this restriction, we shall find in Section III that the analysis filters have very good attenuation characteristics. However, the theoretical development is fairly complicated. We have therefore attempted to make the presentation as complete as possible in order to enable the reader to appreciate the complexity of deriving such QMF bank. Once such a structure is constructed theoretically, its implementation is, however, not nearly as complicated! This is demonstrated at the end of next section by a design example, and in Section IV by an explicit complexity count.

\section{AN LP PR FIR QMF LATTICE STRUCTURE FOR 3-Channel QMF BANK}

From Table I, there are three possibilities here. For one of them, it is easy to see how to decompose $\boldsymbol{E}(z)$ which satisfies the property det $\boldsymbol{E}(z)=b z^{-r}$ under the linearphase constraint. We shall address only this case as the other two appeared to be not easily tractable. Here, we discuss the case where $I=M-1=2$ and $H_{k}(z)$ have the same degrees. Recalling the form for $\boldsymbol{E}(z)$ in (8) which yields LP analysis filters and simplifying it for this particular case, we have

$$
\tilde{E}_{k j}(z)=z^{m_{k}} J_{k} E_{k,(2-j)}(z) ; \quad 0 \leq k \leq 2 .
$$

From (11)

$$
\boldsymbol{P}=\left(\begin{array}{lll}
0 & 0 & 1 \\
0 & 1 & 0 \\
1 & 0 & 0
\end{array}\right)
$$

and thus from ( $15 \mathrm{~b}), \Pi_{k=0}^{2} J_{k}=-1$. Consequently, two analysis filters are symmetric, whereas the remaining one is antisymmetric. Recall that $H_{0}(z)$ and $H_{2}(z)$ are low- pass and high-pass filters, respectively. $H_{0}(z)$ thus, cannot be antisymmetric since antisymmetric LP filters have a zero at $\omega=0$. Likewise, odd-degree symmetric LP filters have a zero of $\omega=\pi$ and, therefore, $H_{2}(z)$ should not be symmetric. Of course, even-degree symmetric $\mathrm{H}_{2}(z)$ would also work; however, it would limit our design to only filters of even length. Consequently, the first two filters, $H_{0}(z)$ and $H_{1}(z)$, are symmetric while $H_{2}(z)$ is antisymmetric. In other words, $J_{0}=J_{1}=1$ and $J_{2}=$ -1 . Writing $\boldsymbol{E}(z)$ from (24) explicitly results in

$$
\boldsymbol{E}(z)=\left(\begin{array}{rrr}
E_{00}(z) & E_{01}(z) & \hat{E}_{00}(z) \\
E_{10}(z) & E_{11}(z) & \hat{E}_{10}(z) \\
E_{20}(z) & E_{21}(z) & -\hat{E}_{20}(z)
\end{array}\right)
$$

where $E_{01}(z)$ and $E_{11}(z)$ are symmetric polynomials, whereas $E_{21}(z)$ is an antisymmetric polynomial. The above $\boldsymbol{E}(z)$ guarantees that the corresponding analysis filters are linear phase. To impose the PR condition, namely, det $\boldsymbol{E}(z)=b z^{-r}$, we decompose $\boldsymbol{E}(z)$ into lower order building blocks as follows:

$$
\boldsymbol{E}(z)=\boldsymbol{E}^{\prime}(z) \boldsymbol{A}(z)
$$

The strategy here is to find $\boldsymbol{A}(z)$ such that $\boldsymbol{E}^{\prime}(z)$ has the same form as $\boldsymbol{E}(z)$. If we continue to decompose $\boldsymbol{E}^{\prime}(z)$ by repeatedly applying (26), we will able to obtain a cascade of building blocks in the form of $\boldsymbol{E}(z)=\boldsymbol{B}(z)$ $\Pi_{i=0}^{L-1} \boldsymbol{A}_{L-1-i}(z)$. For clarification, subscript on $\boldsymbol{A}$ is added. Here, $\boldsymbol{B}$ is the first-order block which has the same form as in (25). This decomposition is not a general way to decompose $\boldsymbol{E}(z)$. In other words, the resulting structure obtained from this decomposition procedure is not guaranteed to cover all triplets of LP PR analysis filters in which $\Pi_{k=0}^{2} J_{k}=-1$ and $I=2$.

Let the elements of $\boldsymbol{E}^{\prime}(z)$ and $\boldsymbol{A}(z)$ be $E_{k l}^{\prime}(z)$ and $A_{k l}(z)$. Then (26) yields (assuming that the orders of $E_{k l}^{\prime}(z)$ are the same and so are the degrees of $\left.A_{k l}(z)\right)$

$$
\begin{gathered}
\left(\begin{array}{rrr}
E_{00}(z) & E_{01}(z) & \hat{E}_{00}(z) \\
E_{10}(z) & E_{11}(z) & \hat{E}_{10}(z) \\
E_{20}(z) & E_{21}(z) & -\hat{E}_{20}(z)
\end{array}\right) \\
=\left(\begin{array}{rrr}
E_{00}^{\prime}(z) & E_{01}^{\prime}(z) & \hat{E}_{00}^{\prime}(z) \\
E_{10}^{\prime}(z) & E_{11}^{\prime}(z) & \hat{E}_{10}^{\prime}(z) \\
E_{20}^{\prime}(z) & E_{21}^{\prime}(z) & -\hat{E}_{20}^{\prime}(z)
\end{array}\right) A(z) .
\end{gathered}
$$

Similarly to $\boldsymbol{E}(z), E_{01}^{\prime}(z)$ and $E_{11}^{\prime}(z)$ are symmetric polynomials, whereas $E_{21}^{\prime}(z)$ is an antisymmetric one. We would like to find the conditions on $A_{k l}(z)$ such that both $\boldsymbol{E}(z)$ and $\boldsymbol{E}^{\prime}(z)$ have the form as in (25). From (27), these 
conditions reflect into the following equations:

$$
\left\{\begin{array}{c}
E_{00}^{\prime}(z)\left(A_{02}(z)-\hat{A}_{20}(z)\right) \\
+E_{01}^{\prime}(z)\left(A_{12}(z)-\hat{A}_{10}(z)\right) \\
=\hat{E}_{00}^{\prime}(z)\left(\hat{A}_{00}(z)-A_{22}(z)\right), \\
E_{10}^{\prime}(z)\left(A_{02}(z)-\hat{A}_{20}(z)\right) \\
+E_{11}^{\prime}(z)\left(A_{12}(z)-\hat{A}_{10}(z)\right) \\
=\hat{E}_{10}^{\prime}(z)\left(\hat{A}_{00}(z)-A_{22}(z)\right), \\
E_{20}^{\prime}(z)\left(A_{02}(z)-\hat{A}_{20}(z)\right) \\
+E_{21}^{\prime}(z)\left(A_{12}(z)-\hat{A}_{10}(z)\right) \\
=-\hat{E}_{20}^{\prime}(z)\left(\hat{A}_{00}(z)-A_{22}(z)\right), \\
E_{00}^{\prime}(z)\left(A_{01}(z)-\hat{A}_{21}(z)\right) \\
+E_{01}^{\prime}(z)\left(A_{11}(z)-\hat{A}_{11}(z)\right) \\
=\hat{E}_{00}^{\prime}(z)\left(\hat{A}_{01}(z)-A_{21}(z)\right), \\
E_{10}^{\prime}(z)\left(A_{01}(z)-\hat{A}_{21}(z)\right) \\
+E_{11}^{\prime}(z)\left(A_{11}(z)-\hat{A}_{11}(z)\right) \\
=\hat{E}_{10}^{\prime}(z)\left(\hat{A}_{01}(z)-A_{21}(z)\right), \\
E_{20}^{\prime}(z)\left(A_{01}(z)-\hat{A}_{21}(z)\right) \\
+E_{21}^{\prime}(z)\left(A_{11}(z)-\hat{A}_{11}(z)\right) \\
=-\hat{E}_{20}^{\prime}(z)\left(\hat{A}_{01}(z)-A_{21}(z)\right) .
\end{array}\right.
$$

We wish to choose $A_{i j}(z)$ such that the above equations hold for any $\boldsymbol{E}^{\prime}(z)$ of the form as in (25). In particular, let $E_{01}^{\prime}(z)=1$ and $E_{00}^{\prime}(z)=0$. From the first and the fourth equations in (28), we have $A_{12}(z)=\hat{A}_{10}(z), A_{11}(z)$ $=\hat{A}_{11}(z)$. Using these relations, $(28)$ is reduced to

$$
\left\{\begin{array}{c}
E_{00}^{\prime}(z)\left(A_{02}(z)-\hat{A}_{20}(z)\right) \\
=\hat{E}_{00}^{\prime}(z)\left(\hat{A}_{00}(z)-A_{22}(z)\right), \\
E_{10}^{\prime}(z)\left(A_{02}(z)-\hat{A}_{20}(z)\right) \\
=\hat{E}_{10}^{\prime}(z)\left(\hat{A}_{00}(z)-A_{22}(z)\right), \\
E_{20}^{\prime}(z)\left(A_{02}(z)-\hat{A}_{20}(z)\right) \\
=-\hat{E}_{20}^{\prime}(z)\left(\hat{A}_{00}(z)-A_{22}(z)\right), \\
E_{00}^{\prime}(z)\left(A_{01}(z)-\hat{A}_{21}(z)\right) \\
=\hat{E}_{00}^{\prime}(z)\left(\hat{A}_{01}(z)-A_{21}(z)\right), \\
E_{10}^{\prime}(z)\left(A_{01}(z)-\hat{A}_{21}(z)\right) \\
=\hat{E}_{10}^{\prime}(z)\left(\hat{A}_{01}(z)-A_{21}(z)\right), \\
E_{20}^{\prime}(z)\left(A_{01}(z)-\hat{A}_{21}(z)\right) \\
=-\hat{E}_{20}^{\prime}(z)\left(\hat{A}_{01}(z)-A_{21}(z)\right) .
\end{array}\right.
$$

To find the corresponding relations of the remaining $A_{i j}(z)$, let $E_{00}^{\prime}(z)=E_{20}^{\prime}(z)=1$. This choice of $E^{\prime}(z)$ will yield $A_{20}(z)=\hat{A}_{02}(z), A_{22}(z)=\hat{A}_{00}(z)$ and $A_{21}(z)=$ $\hat{A}_{01}(z)$. In summary, $A_{i j}(z)$ has to satisfy the following conditions if (28) holds for all choices of $\boldsymbol{E}^{\prime}(z)$ of the form as in $(25)$

$$
\begin{cases}A_{20}(z)=\hat{A}_{02}(z), & A_{12}(z)=\hat{A}_{10}(z), \\ A_{22}(z)=\hat{A}_{00}(z), & A_{21}(z)=\hat{A}_{01}(z), \\ A_{11}(z)=\hat{A}_{11}(z) . & \end{cases}
$$

$\boldsymbol{A}(z)$ then takes the form

$$
\boldsymbol{A}(z)=\left(\begin{array}{ccc}
A_{00}(z) & A_{01}(z) & \hat{A}_{20}(z) \\
A_{10}(z) & A_{11}(z) & \hat{A}_{10}(z) \\
A_{20}(z) & \hat{A}_{01}(z) & \hat{A}_{00}(z)
\end{array}\right)
$$

where $A_{11}(z)$ is symmetric. Continuing the decomposition process and putting subscript on $\boldsymbol{A}(z)$, we see that $\boldsymbol{E}(z)$ is realized as a cascade of lower order building blocks, i.e.,

$$
\boldsymbol{E}(z)=\boldsymbol{B}(z) \prod_{i=0}^{L-1} \boldsymbol{A}_{L-1-i}(z)
$$

where $L$ is the number of $\boldsymbol{A}_{i}(z)$ blocks in the structure. The analysis bank in Fig. 2 thus becomes Fig. 4 where $\boldsymbol{A}_{i}(z)$ is as in (30) and $\boldsymbol{B}(z)$ has the same form as in (25). We now can impose the PR condition $\operatorname{det} \boldsymbol{E}(z)=z^{-r}$ on each building block so that the overall structure is a PR system.

The simplest $\boldsymbol{B}(z)$ which satisfies simultaneously (25) and $\operatorname{det} \boldsymbol{B}(z)=z^{-r}$ is

$$
\boldsymbol{B}=\left(\begin{array}{rrr}
1 & 0 & 1 \\
0 & 1 & 0 \\
1 & 0 & -1
\end{array}\right)
$$

Since the above choice for $\boldsymbol{B}(z)$ is a constant matrix, $\boldsymbol{A}_{i}(z)$ must be a function of $z$. From (30)

$$
\boldsymbol{A}_{i}(z)=\Gamma_{3} \hat{A}_{i}(z) \Gamma_{3}
$$

where $\Gamma_{3}$ is defined in (12). Let the highest degrees of the elements $A_{i, k l}(z)$ of $A_{i}(z)$ be $r_{1}$, and let the determinant of $\boldsymbol{A}_{i}(z)$ be $z^{-r 2}$. We would like to find the minimum values of both $r_{1}$ and $r_{2}$ such that (33) is satisfied. Taking the determinant of both sides in (33), we obtain

$$
\begin{aligned}
\operatorname{det} \boldsymbol{A}_{i}(z) & =\operatorname{det} \hat{\boldsymbol{A}_{i}}(z)=\operatorname{det}\left[z^{-r_{1}} \boldsymbol{A}_{i}\left(z^{-1}\right)\right] \\
z^{-r_{2}} & =z^{-3 r_{1}+r_{2}} .
\end{aligned}
$$

Thus, the minimum values for both $r_{1}$ and $r_{2}$ are $r_{1}=2$ and $r_{2}=3$, respectively. Consider the following form for $\boldsymbol{A}_{i}(z)$ :

$$
\boldsymbol{A}_{i}(z)=U_{i} \mathbf{\Lambda}(z) \boldsymbol{V}_{i}
$$




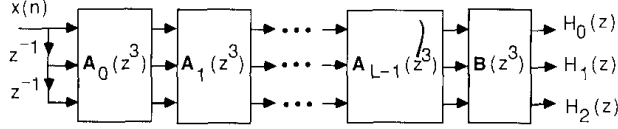

Fig. 4. Decomposition of $E\left(z^{3}\right)$ into a cascade of building blocks.

where $U_{i}$ and $V_{i}$ are nonsingular matrices and

$$
\boldsymbol{\Lambda}(z)=\left(\begin{array}{lll}
1 & 0 & 0 \\
0 & z^{-1} & 0 \\
0 & 0 & z^{-2}
\end{array}\right)
$$

Clearly, det $\boldsymbol{\Lambda}(z)=z^{-3}$. To satisfy (33), $\boldsymbol{U}_{i}$ and $\boldsymbol{V}_{i}$ have to take special forms. Substituting (34) into (33), $\boldsymbol{U}_{i}$ and $V_{i}$ satisfy

$$
\left\{\begin{array}{l}
\boldsymbol{U}_{i}=\Gamma_{3} \boldsymbol{U}_{i} \Gamma_{3}, \\
\boldsymbol{V}_{i}=\Gamma_{3} \boldsymbol{V}_{i} \Gamma_{3} .
\end{array}\right.
$$

In other words, both $U_{i}$ and $V_{i}$ have the form

$$
\boldsymbol{U}_{i}=V_{i}=\left(\begin{array}{ccc}
1 & a_{i} & b_{i} \\
c_{i} & d_{i} & c_{i} \\
b_{i} & a_{i} & 1
\end{array}\right)
$$

With the above $\boldsymbol{A}_{i}(z)$ and $\boldsymbol{B}, \boldsymbol{E}(z)$ becomes

$$
\begin{aligned}
\boldsymbol{E}(z)= & B \boldsymbol{U}_{L-1} \Lambda(z) V_{L-1} U_{L-2} \Lambda(z) V_{L-2} \\
& \cdots U_{0} \Lambda(z) V_{0} .
\end{aligned}
$$

Observing from (37), $\boldsymbol{V}_{i} \boldsymbol{U}_{j}$ satisfies $\boldsymbol{V}_{i} \boldsymbol{U}_{j}=\Gamma_{3} \boldsymbol{V}_{i} \boldsymbol{U}_{j} \Gamma_{3}$, and has the same form as $U_{i}$ in addition to being a nonsingular matrix. Therefore, we can use the general symbol $W_{i}$ for $U_{i}, V_{i}$ and $V_{i} U_{j}$. The polyphase transfer matrix $\boldsymbol{E}(z)$ in (38) is equivalent to

$$
\boldsymbol{E}(z)=\boldsymbol{B} \boldsymbol{W}_{L-1} \Lambda(z) \boldsymbol{W}_{L-2} \boldsymbol{\Lambda}(z) \cdots \boldsymbol{W}_{1} \boldsymbol{\Lambda}(z) \boldsymbol{W}_{0}
$$

where

$$
\boldsymbol{W}_{i}=\left(\begin{array}{ccc}
1 & a_{i} & b_{i} \\
c_{i} & d_{i} & c_{i} \\
b_{i} & a_{i} & 1
\end{array}\right)
$$

Fig. 4 thus becomes Fig. 5 where $\boldsymbol{B}, \boldsymbol{\Lambda}(z)$, and $\boldsymbol{W}_{i}$ are as in (32), (35), and (40). Assuming that $d_{i} \neq 0$, the above $W_{i}$ can be factorized as

$$
W_{i}=\left(\begin{array}{lll}
1 & 0 & 0 \\
0 & d_{i} & 0 \\
0 & 0 & 1
\end{array}\right)\left(\begin{array}{lll}
1 & a_{i} & b_{i} \\
c_{i} / d_{i} & 1 & c_{i} / d_{i} \\
b_{i} & a_{i} & 1
\end{array}\right)
$$

Furthermore, we notice that the multiplier $d_{i}$ can be propagated through the entire analysis bank and can be grouped into the multiplier $\beta_{2}$ at the end of Fig. 5. By propagating

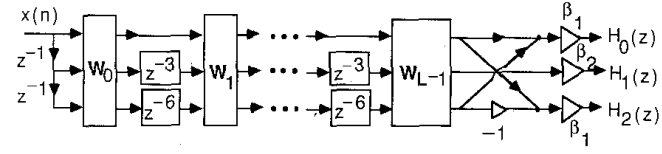

Fig. 5. The LP PR analysis bank.

$d_{i}$ through the analysis bank, the multipliers $a_{i}, b_{i}$, and $c_{i}$ in $W_{i}$ are changed. The building block $W_{i}$ in Fig. 5 thus takes the following form:

$$
W_{i}=\left(\begin{array}{ccc}
1 & a_{i} & b_{i} \\
c_{i} & 1 & c_{i} \\
b_{i} & a_{i} & 1
\end{array}\right)
$$

where the set of lattice coefficients $a_{i}, b_{i}, c_{i}$ is different from the one in (40). In summary, Fig. 5 is the analysis bank of an LP PR FIR QMF structure which yields $H_{k}(z)$ of the same degrees. Moreover, $H_{0}(z)$ and $H_{1}(z)$ are symmetric, whereas $H_{2}(z)$ is antisymmetric. The degree of $H_{k}(z), 0 \leq k \leq 2$ is $6 L-4$ where $L$ is the number of $W_{i}(z)$ blocks in the analysis bank.

Without loss of generality, let us assume that $H_{0}(z)$, $H_{1}(z)$, and $H_{2}(z)$ are low-pass, bandpass, and high-pass filters, respectively. To design $H_{k}(z)$, we define an objective function which represents both the stopband and passband errors as follows:

$$
\begin{aligned}
\Phi_{1}= & \sum_{k=0}^{2} \int_{k^{\text {th stopband }}}\left|H_{k}\left(e^{j \omega}\right)\right|^{2} d \omega \\
& +\sum_{k=0}^{2} \int_{k^{\text {th }} \text { passband }}\left|z^{\left(N_{k}-1\right) / 2}-H_{k}\left(e^{j \omega}\right)\right|^{2} d \omega .
\end{aligned}
$$

The optimization of the parameters of $\boldsymbol{W}_{i}$ (so as to minimize $\Phi_{1}$ ) can be done by employing standard gradient algorithms [21]. This usually consumes time since the objective function $\Phi_{1}$ is a nonlinear function of many parameters. Suppose that $H_{k}(z)$ has pairwise-symmetry property [19], i.e.,

$$
H_{2}(z)=H_{0}(-z), \quad H_{1}(z)=\alpha_{1}\left(z^{2}\right)
$$

for some $\alpha_{1}(z)$. It is shown in [19] that the structure presented in Fig. 9 of [19] yields filters which satisfy the pairwise symmetry condition for odd $M$. For $M=3$, Fig. 9 of [19] simplifies to Fig. 6 where

$$
\boldsymbol{E}_{1}(z)=\boldsymbol{W}_{L-1} \boldsymbol{\Lambda}(z) \boldsymbol{W}_{L-2} \cdots \boldsymbol{\Lambda}(z) \boldsymbol{W}_{0} .
$$

In other words, the structure in Fig. 6 is an analysis bank of an LP PR FIR QMF structure. Furthermore, $H_{k}(z)$ satisfies the pairwise-symmetric property described above. Since $\boldsymbol{E}_{1}(z)$ is a function of $z^{6}$ instead of $z^{3}$ as in Fig. 5, for a given order of the analysis filter, the structure in Fig. 6 has approximately half the number of variables compared to the structure in Fig. 5. Consequently, the convergence of the optimization using pairwise-symmetric structure will be much faster than the one in Fig. 5. 


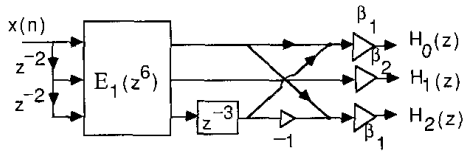

Fig. 6. The pairwise symmetric LP PR analysis bank.

With the above pairwise-symmetry property enforced in the structure, it is now sufficient to optimize

$$
\begin{aligned}
\Phi_{2}= & \int_{(\pi / 3)+\epsilon}^{\pi}\left|H_{0}\left(e^{j \omega}\right)\right|^{2} d \omega \\
& +\int_{0}^{(\pi / 3)-\epsilon}\left|z^{\left(N_{0}-1\right) / 2}-H_{0}\left(e^{j \omega}\right)\right|^{2} d \omega \\
& +\int_{0}^{(\pi / 3)-\epsilon}\left|H_{1}\left(e^{j \omega}\right)\right|^{2} d \omega \\
& +\int_{(\pi / 3)+\epsilon}^{\pi / 2}\left|z^{\left(N_{1}-1\right) / 2}-H_{1}\left(e^{j \omega}\right)\right|^{2} d \omega
\end{aligned}
$$

where $\epsilon$ depends on the desired stopband edges. Therefore, if we can impose the pairwise-symmetry condition on the structure of Fig. 5, then we would save approximately half of the computation time in terms of the stopbands and passband error-computations in the objective function. Thus, together with the saving in the number of variables to be optimized, we would expect a faster convergence time for the pairwise symmetric LP PR structure in Fig. 6.

Comments on the Synthesis Filters $F_{k}(z)$ : The synthesis filters $F_{k}(z)$ of the PR structure are obtained as

$$
F_{k}(z)=\sum_{l=0}^{2} z^{-(2-l)} R_{l k}\left(z^{3}\right)
$$

where $\boldsymbol{R}(z)=\boldsymbol{E}^{-1}(z)$. By computing $\boldsymbol{E}^{-1}(z)$ explicitly, we will show that $F_{k}(z)$ are also linear-phase filters of the same symmetries as that of $H_{k}(z)$. Calculating $\boldsymbol{R}(z)$ from (25), we observe that $R_{l k}(z)$ satisfies the condition

$$
\tilde{R}_{l k}(z)=z^{m_{k}} J_{k} R_{(2-l), k}(z)
$$

which has the same form as in (24) (except for the transposition). Thus, $F_{k}(z)$ is also linear phase of the same type of symmetry as $H_{k}(z)$. By inverting the analysis bank in Figs. 5 and 6 , we will conclude below that $F_{k}(z)$ has the same degree as $H_{k}(z)$.

In general, $F_{k}(z)$ has higher degree compared to $H_{k}(z)$ due to the inversion process of $\boldsymbol{E}(z)$. Hence, if we implement the analysis bank as in (39), then

$$
\boldsymbol{R}(z)=\boldsymbol{W}_{0}^{-1} \boldsymbol{\Lambda}^{-1}(z) \boldsymbol{W}_{1}^{-1} \cdots \boldsymbol{\Lambda}^{-1}(z) \boldsymbol{W}_{L-1}^{-1} \boldsymbol{B}^{-1} .
$$

By noting that $\boldsymbol{\Lambda}^{-1}(z)=z^{-2} \Gamma_{3} \boldsymbol{\Lambda}(z) \Gamma_{3}$, the corresponding synthesis bank of Fig. 5 is drawn in Fig. 7. It is clear from Fig. 7 that, in this particular instance, $F_{k}(z)$ actually has the same degree as $H_{k}(z)$. Further conclusions can be drawn for the synthesis filters corresponding to the analysis bank in Fig. 6, namely, the synthesis filters also satisfy the pairwise symmetric property. In other words,

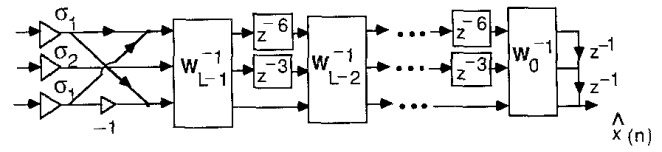

Fig. 7. The synthesis bank of Fig. 5. Here $\sigma_{1}=1 /\left(\beta_{1} \sqrt{2}\right)$ and $\sigma_{2}=1 / \beta_{2}$.

$F_{2}(z)=-F_{0}(-z)$ and $F_{1}(z)=z^{-3} \alpha_{2}\left(z^{2}\right)$ for some function $\alpha_{2}(z)$. In short, if we implement the analysis filters as in Figs. 5 or 6 , then the synthesis filters are also linear phase with the same corresponding symmetries as $H_{k}(z)$, and their degrees are the same as that of $H_{k}(z)$.

Comments on the LP PR Pairwise Symmetry Analysis Bank of Fig. 6: Fig. 6 is an LP PR analysis bank which yields pairwise symmetric analysis filters. We obtain this structure by using $E_{1}(z)$ in (42) with $z$ replaced by $z^{2}$. The degrees of $H_{0}(z), H_{1}(z)$, and $H_{2}(z)$ are thus ( $12 L-$ $5),(12 L-8)$, and $(12 L-5)$, respectively. In addition to the pairwise-symmetric property, namely, $h_{2}(n)=$ $(-1)^{n} h_{0}(n)$ and $h_{1}(n)=0$ for even $n$, the coefficients of $H_{k}(z)$ also satisfy

$\left\{\begin{array}{l}h_{0}(1)=h_{0}(12 L-6)=h_{2}(1)=h_{2}(12 L-6)=0, \\ h_{1}(n)=0, \quad n \text { odd. }\end{array}\right.$

Using the above fact to compute the degrees of $E_{k j}(z)$, we have

$$
\operatorname{deg}[\boldsymbol{E}(z)]=\left(\begin{array}{lll}
4 L-3 & 4 L-2 & 4 L-3 \\
4 L-4 & 4 L-3 & 4 L-4 \\
4 L-3 & 4 L-2 & 4 L-3
\end{array}\right)
$$

From the above lengths of $H_{k}(z), I=1$ instead of $I=2$ as in the structure of Fig. 5. Moreover, the lengths of $H_{k}(z)$ are not the same. This, however, is not surprising since we have taken an LP PR structure in which $I=2$ and $N_{k}$ are the same, and have transformed it into a structure in which $I=1$ and $N_{k}$ are not the same by imposing the pairwise-symmetric property.

Example 3.1: Using the structure in Fig. 6 and taking the number of $\boldsymbol{W}_{i}$ blocks to be $L=5$, we design $H_{k}(z)$ for $\epsilon=0.1 \pi$. The degrees of the analysis filters are 55, 52 , and 55, respectively. The 15 variables in the lattice structure and the 2 additional multipliers at the output were optimized using the IMSL subroutine [21] on a computer to minimize (43). The resulting frequency response magnitudes are shown in Fig. 8(a). The lattice coefficients and impulse responses of $H_{k}(z)$ are given in Tables II and III, respectively. Table III only displays half the number of coefficients of $H_{k}(z)$ since they are linear-phase filters. The pairwise-symmetry property is apparent in Table III. The frequency response magnitudes of the synthesis filters associated with the analysis filters in Example 3.1 are shown in Fig. 8(b). We display only half the number of coefficients of $F_{k}(z)$ in Table IV since $F_{k}(z)$ are linearphase filters. 


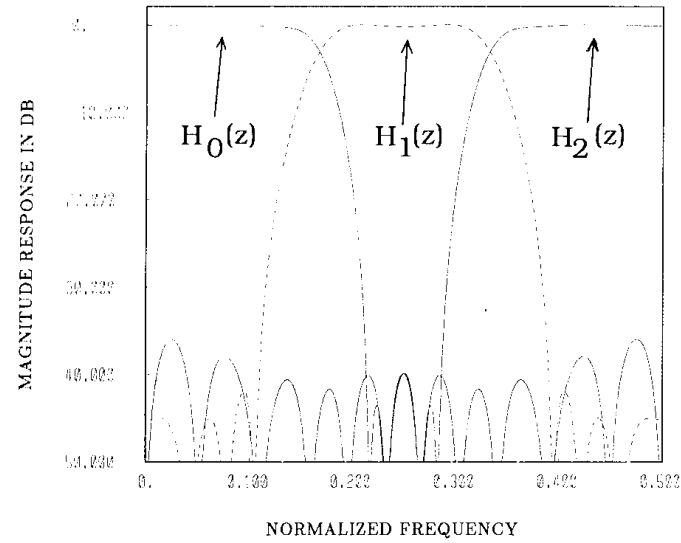

(a)

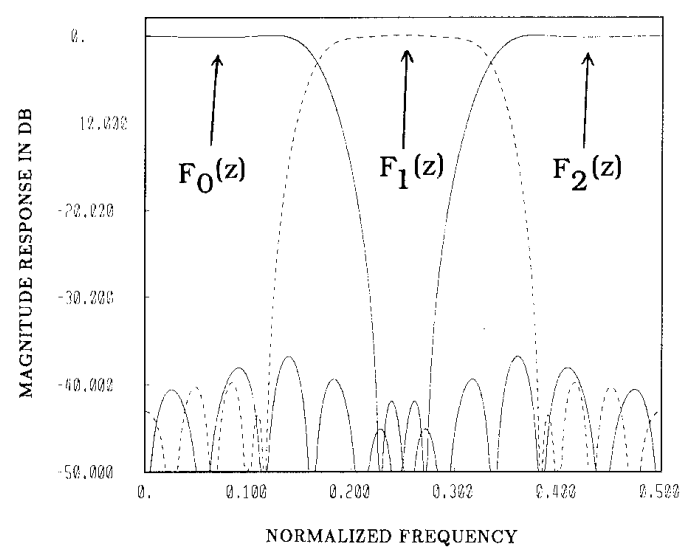

(b)

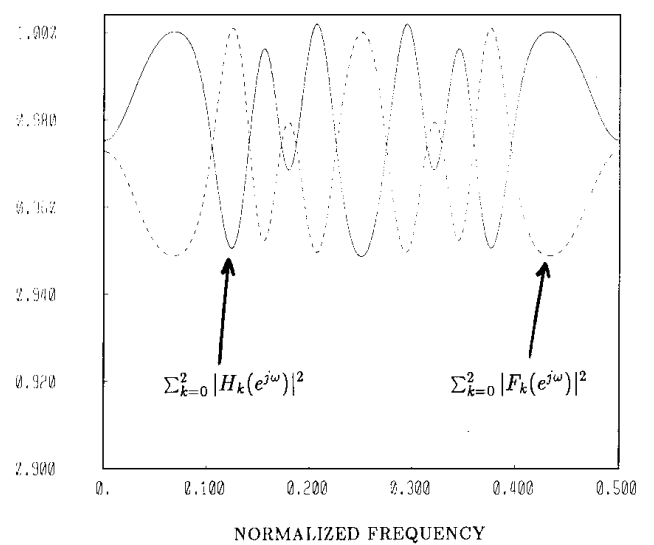

(c)

Fig. 8. (a) Example 3.1: Magnitude response plots for the optimized analysis filters. (b) Example 3.1: Magnitude response plots for the corre sponding synthesis filters. (c) Example 3.1: The plots of $\Sigma_{k=0}^{2}\left|H_{k}\left(e^{j \omega}\right)\right|^{2}$ (solid line) and $\Sigma_{k=0}^{2}\left|F_{k}\left(e^{j \omega}\right)\right|^{2}$ (broken line).

In most of the earlier designs of PR systems [12], [15], [19], the LBR condition is enforced on the structure, and consequently the analysis filters satisfied the power-complementary property, i.e., $\Sigma_{k=0}^{M-1},\left.H_{k}\left(e^{j \omega}\right)\right|^{2}=1$. How-
TABLE II

Lattice Coefficients of the Optimized Analysis Bank in Example 3.1. $\beta_{1}=-4.1034794220864 \times 10^{-9}$ AND $\beta_{2}=1.5707941418142 \times$ $10^{-1}$

\begin{tabular}{c||r|r|r}
\hline \hline$m$ & Lattice Coefficients $a_{m}$ & Lattice Coefficients $b_{m}$ & Lattice Coefficients $c_{m}$ \\
\hline 0 & $-2.3282102148565 \times 10^{3}$ & $-5.5293447341746 \times 10^{3}$ & $6.1485544476533 \times 10^{-1}$ \\
\hline 1 & $5.4771339365037 \times 10^{4}$ & $-1.7872270513189 \times 10^{2}$ & $-1.9882974847877 \times 10^{-5}$ \\
\hline 2 & $-1.3351781493993 \times 10^{6}$ & $1.3096283152688 \times 10^{0}$ & $-7.4289672830093 \times 10^{-7}$ \\
\hline 3 & $-8.6710986482818 \times 10^{6}$ & $4.3087219824673 \times 10^{1}$ & $-2.1443436695392 \times 10^{-8}$ \\
\hline 4 & $-3.7906401528742 \times 10^{8}$ & $-6.8094743277621 \times 10^{0}$ & $-6.3599130248406 \times 10^{-6}$ \\
\hline
\end{tabular}

TABLE III

Impulse Responses of the Optimized Analysis Filters in Example 3.1. Here $N_{0}-1=55$ AND $N_{1}-1=52$. FURTHERMORE, $h_{0}(m)=$ $h_{0}\left(N_{0}-1-m\right), h_{1}(m)=h_{1}\left(N_{1}-1-m\right)$ AND $h_{2}(m)=(-1)^{m} h_{0}(m)$

\begin{tabular}{c||r|r|r}
\hline \hline$m$ & Filter Coefficients $h_{0}(\mathrm{~m})$ & Filter Coefficients $h_{1}(\mathrm{~m})$ & Filter Coefficients $h_{2}(\mathrm{~m})$ \\
\hline 0 & $-4.1034799150810 \times 10^{-9}$ & $-9.9901150220313 \times 10^{-9}$ & $-4.1034799150810 \times 10^{-9}$ \\
\hline 1 & 0 & 0. & 0 \\
\hline 2 & $9.5537638547501 \times 10^{-6}$ & $2.3259087841885 \times 10^{-5}$ & $9.5537638547501 \times 10^{-6}$ \\
\hline 3 & $2.7942540296973 \times 10^{-8}$ & 0 & $-2.7942540296973 \times 10^{-8}$ \\
\hline 4 & $2.2689555060244 \times 10^{-5}$ & $5.5238789890867 \times 10^{-5}$ & $2.2689555060244 \times 10^{-5}$ \\
\hline 5 & $-6.5056107748452 \times 10^{-5}$ & 0 & $6.5056107748452 \times 10^{-5}$ \\
\hline 6 & $-1.3835938232415 \times 10^{-4}$ & $-3.3676459377498 \times 10^{-4}$ & $-1.3835938232415 \times 10^{-4}$ \\
\hline 7 & $-1.5450393805053 \times 10^{-4}$ & 0. & $1.5450393805053 \times 10^{-4}$ \\
\hline 8 & $1.6807358248882 \times 10^{-4}$ & $2.2796523469311 \times 10^{-4}$ & $1.6807358248882 \times 10^{-4}$ \\
\hline 9 & $9.4189414966001 \times 10^{-4}$ & 0 & $-9.4189414966001 \times 10^{-4}$ \\
\hline 10 & $7.9474660355847 \times 10^{-4}$ & $1.5044677383506 \times 10^{-3}$ & $7.9474660355847 \times 10^{-4}$ \\
\hline 11 & $-5.3803123943823 \times 10^{-4}$ & 0 & $5.3803123943823 \times 10^{-4}$ \\
\hline 12 & $-2.7006977979884 \times 10^{-3}$ & $-3.9523449509934 \times 10^{-3}$ & $-2.7006977979884 \times 10^{-3}$ \\
\hline 13 & $-3.9715006886561 \times 10^{-3}$ & 0. & $3.9715006886561 \times 10^{-3}$ \\
\hline 14 & $2.3956889217717 \times 10^{-3}$ & $6.8329546163215 \times 10^{-3}$ & $2.3956889217717 \times 10^{-3}$ \\
\hline 15 & $9.6102366520721 \times 10^{-3}$ & 0. & $-9.6102366520721 \times 10^{-3}$ \\
\hline 16 & $5.8659326584867 \times 10^{-3}$ & $9.1535965074864 \times 10^{-3}$ & $5.8659326584867 \times 10^{-3}$ \\
\hline 17 & $-1.2145874803718 \times 10^{-2}$ & 0. & $1.2145874803718 \times 10^{-2}$ \\
\hline 18 & $-1.5561448572774 \times 10^{-2}$ & $-4.7294831413144 \times 10^{-2}$ & $-1.5561448572774 \times 10^{-2}$ \\
\hline 19 & $-4.9352298948535 \times 10^{-3}$ & 0. & $4.9352298948535 \times 10^{-3}$ \\
\hline 20 & $2.4192899584657 \times 10^{-2}$ & $3.4028581273034 \times 10^{-2}$ & $2.4192899584657 \times 10^{-2}$ \\
\hline 21 & $2.8655843071586 \times 10^{-2}$ & 0. & $-2.8655843071586 \times 10^{-2}$ \\
\hline 22 & $-6.8884118701520 \times 10^{-3}$ & $9.4252950512146 \times 10^{-2}$ & $-6.8884118701520 \times 10^{-3}$ \\
\hline 23 & $-5.7672278487990 \times 10^{-2}$ & 0. & $5.7672278487990 \times 10^{-2}$ \\
\hline 24 & $-5.6803993889263 \times 10^{-2}$ & $-2.8879891452761 \times 10^{-1}$ & $-5.6803993889263 \times 10^{-2}$ \\
\hline 25 & $4.3175380598407 \times 10^{-2}$ & 0. & $-4.3175380598407 \times 10^{-2}$ \\
\hline 26 & $2.0762730202796 \times 10^{-1}$ & $3.8301848634403 \times 10^{-1}$ & $2.0762730202796 \times 10^{-1}$ \\
\hline 27 & $3.3497547410063 \times 10^{-1}$ & & $-3.3497547410063 \times 10^{-1}$ \\
\hline & & &
\end{tabular}

ever, the LBR condition is not necessary for PR systems, and as demonstrated in Fig. 8(c) for our design example, $\Sigma_{k=0}^{2}\left|H_{k}\left(e^{j \omega}\right)\right|^{2} \neq 1$ (solid line) and $\Sigma_{k=0}^{2}\left|F_{k}\left(e^{j \omega}\right)\right|^{2} \neq$ 1 (broken line). In other words, the filters $H_{k}(z)$ are not power-complementary triplets.

Implementation of this system on a fixed-point machine might require a very large number of bits because of the large dynamic range spanned by the coefficients in Tables II and III. However, a single precision floating-point implementation was found to be very satisfactory in this case. In order to demonstrate the perfect-reconstruction property of the QMF bank characterized by the impulse responses in Tables III and IV, and by the lattice coefficients in Table II, the complete systems of Fig. 1 (directform) and of Fig. 6 (lattice-form) were simulated in Fortran on a VAX 11/750 machine using both single and double precisions. Table $\mathrm{V}$ shows an arbitrary input $x(n)$ and the reconstructed signal $\hat{x}(n)$. It is clear that the system has a perfect-reconstruction property except for roundoff errors. From the double-precision implementation, the lattice structure seems to be numerically much more robust than the direct form. 
TABLE IV

Impulse Responses of the Synthesis Filters in Examples 3.1. Here $N_{0}^{\prime}$ $-1=55$, AND $N_{1}^{\prime}-1=58$. FuRTHERMORE, $f_{0}(m)=f_{0}\left(N_{0}^{\prime}-1-m\right)$ $f_{1}(m)=f_{1}\left(N_{1}^{\prime}-1-m\right)$ AND $f_{2}(m)=(-1)^{(m+1)} f_{0}(m)$

\begin{tabular}{|c|c|c|c|}
\hline$m$ & Filter Coefficients $f_{0}(m)$ & Filter Coefficients $f_{1}(m)$ & Filter Coefficients $f_{2}(m)$ \\
\hline 0 & $-5.0856051950609 \times 10^{-7}$ & 0 & $5.0856051950609 \times 10^{-7}$ \\
\hline 1 & - 0. & 0. & 0 \\
\hline 2 & $1.2071726295669 \times 10^{-6}$ & 0. & $-1.2071726295669 \times 10^{-6}$ \\
\hline 3 & $6.8043026031286 \times 10^{-7}$ & $-3.4038856600144 \times 10^{-6}$ & $6.8043026031286 \times 10^{-7}$ \\
\hline 4 & $-1.4547832873095 \times 10^{-6}$ & 0 & $1.4547832873095 \times 10^{-6}$ \\
\hline & $-1.6151406864545 \times 10^{-6}$ & $8.0798202875348 \times 10^{-6}$ & $-1.6151406864545 \times 10^{-6}$ \\
\hline 6 & $-1.0954083283872 \times 10^{-4}$ & 0. & $1.0954083283872 \times 10^{-4}$ \\
\hline 7 & $1.9464322001326 \times 10^{-6}$ & $-9.7371222896162 \times 10^{-6}$ & $1.9464322001326 \times 10^{-6}$ \\
\hline 8 & $2.6002386734911 \times 10^{-4}$ & 0 & $-2.6002386734911 \times 10^{-4}$ \\
\hline 9 & $1.3510398808581 \times 10^{-4}$ & $-7.2376456380997 \times 10^{-1}$ & $1.3510398808581 \times 10^{-4}$ \\
\hline 10 & $-2.9880316517939 \times 10^{-4}$ & 0. & $2.9880316517939 \times 10^{-4}$ \\
\hline 11 & $-3.2070536761859 \times 10^{-1}$ & $1.7180453614011 \times 10^{-3}$ & $-3.2070536761859 \times 10^{-4}$ \\
\hline 12 & $-1.1666078385066 \times 10^{-3}$ & 0. & $1.1666078385066 \times 10^{-3}$ \\
\hline 13 & $3.6701223936154 \times 10^{-4}$ & $-1.9730196839034 \times 10^{-3}$ & $3.6701223936154 \times 10^{-4}$ \\
\hline 14 & $2.5801957392175 \times 10^{-3}$ & 0 & $-2.5801957392175 \times 10^{-3}$ \\
\hline$\overline{15}$ & $2.8033633714558 \times 10^{-3}$ & $-8.4270347872648 \times 10^{-3}$ & $2.8033633714558 \times 10^{-3}$ \\
\hline 16 & $1.6293136197350 \times 10^{-3}$ & 0. & $-1.6293136197350 \times 10^{-3}$ \\
\hline 17 & $-6.4013608859602 \times 10^{-3}$ & $1.8738254668239 \times 10^{-2}$ & $-6.4013608859602 \times 10^{-3}$ \\
\hline 18 & $-1.4974601249272 \times 10^{-2}$ & 0 & $1.4974601249272 \times 10^{-2}$ \\
\hline 19 & $1.7020623944998 \times 10^{-3}$ & $8.8661384068888 \times 10^{-3}$ & $1.7020623944998 \times 10^{-3}$ \\
\hline 20 & $2.2605984154776 \times 10^{-2}$ & 0. & $-2.2605984154776 \times 10^{-2}$ \\
\hline 21 & $2.9538667731008 \times 10^{-2}$ & $-4.8496302353281 \times 10^{-2}$ & $2.9538667731008 \times 10^{-2}$ \\
\hline 22 & $-4.7736225613466 \times 10^{-3}$ & 0. & $4.7736225613466 \times 10^{-3}$ \\
\hline 23 & $-5.7106872050697 \times 10^{-2}$ & $3.2040864597603 \times 10^{-2}$ & $-5.7106872050697 \times 10^{-2}$ \\
\hline 24 & $-5.8850606121214 \times 10^{-2}$ & 0. & $5.8850606121214 \times 10^{-2}$ \\
\hline 25 & $3.9318475835318 \times 10^{-2}$ & $9.9810904531240 \times 10^{-2}$ & $3.9318475835318 \times 10^{-2}$ \\
\hline 26 & $2.0744767026860 \times 10^{-1}$ & 0 & $-2.0744767026860 \times 10^{-1}$ \\
\hline 27 & $3.3879421 .236868 \times 10^{-1}$ & $-2.8455714941648 \times 10^{-1}$ & $3.3879421236868 \times 10^{-1}$ \\
\hline 28 & & 0. & \\
\hline & & $3.7311674030318 \times 10^{-1}$ & \\
\hline
\end{tabular}

TABLE V

An ARbitrary InPUt Sequence $x(n)$ and the Reconstructed Signal $\hat{x}(n)$ FOR THE Design EXAmple. Here $\hat{x}(n+N-1)$ is SHOWN IN ORder TO ALIGN THE SAMPLES

\begin{tabular}{c|c|c|c|c|c}
\hline$n$ & $x(n)$ & $\begin{array}{c}\hat{x}(n+N-1) \\
\text { Direct Form } \\
\text { Single precision }\end{array}$ & $\begin{array}{c}\hat{x}(n+N-1) \\
\text { Direct Form } \\
\text { Double precision }\end{array}$ & $\begin{array}{c}\hat{x}(n+N-1) \\
\text { Lattice Structure } \\
\text { Single precision }\end{array}$ & $\begin{array}{c}\hat{x}(n+N-1) \\
\text { Lattice Structure } \\
\text { Double precision }\end{array}$ \\
\hline 0 & 1.00000 & 1.00000 & 1.0000012161693 & 1.00000 & 1.0000000000000 \\
\hline 1 & 2.00000 & 2.00000 & 1.9999999838918 & 2.00000 & 2.0000000000000 \\
\hline 2 & 3.00000 & 3.00000 & 3.0000013029297 & 3.00000 & 3.0000000000000 \\
\hline 3 & 4.00000 & 4.00000 & 4.0000016419205 & 4.00000 & 4.0000000000000 \\
\hline 4 & 5.00000 & 5.00000 & 5.0000003711781 & 5.00000 & 5.0000000000000 \\
\hline 5 & 6.00000 & 6.00000 & 6.0000033301603 & 6.00000 & 6.0000000000000 \\
\hline 6 & 7.00000 & 7.00000 & 7.0000020906728 & 7.00000 & 7.0000000000000 \\
\hline 7 & 8.00000 & 8.00000 & 8.0000011335777 & 7.99999 & 8.0000000000000 \\
\hline 8 & 9.00000 & 9.00000 & 9.0000045952801 & 9.00000 & 9.0000000000000 \\
\hline 9 & 10.00000 & 10.00000 & 10.000000041374 & 10.00000 & 10.0000000000000 \\
\hline 10 & 11.00000 & 11.00000 & 11.000001869694 & 11.00000 & 11.0000000000000 \\
\hline
\end{tabular}

Note that single precision corresponds to 24 bits of mantissa and 8 bits of exponent, which is identical to the arithmetic operations in the AT\&T DSP 32 signal processor. The conclusion is that the perfect-reconstruction system can be implemented on such a commercial DSP chip easily, and the fact that the filter coefficients span a large dynamic range is immaterial in such implementations.

Implementation of the PR System: From (41)

$$
W_{i}^{-1}=\frac{1}{\Delta_{i}}\left(\begin{array}{lll}
1-a_{i} c_{i} & a_{i}\left(b_{i}-1\right) & a_{i} c_{i}-b_{i} \\
c_{i}\left(b_{i}-1\right) & 1-b_{i}^{2} & c_{i}\left(b_{i}-1\right) \\
a_{i} c_{i}-b_{i} & a_{i}\left(b_{i}-1\right) & 1-a_{i} c_{i}
\end{array}\right),
$$

where $\Delta_{i}=\left(1-b_{i}\right)\left(1+b_{i}-2 a_{i} c_{i}\right)$. Let us use $b$ bits to implement each multiplier in $W_{i}$. It appears at first that we would require infinite precision to implement each multiplier in $\boldsymbol{W}_{i}^{-1}$ since it involves a division by $\Delta_{i}$. These terms $\Delta_{i}$, however, can be grouped with the multipliers $\beta_{i}$ at the end of the structure. Each multiplier in the above $\boldsymbol{W}_{i}^{-1}$ thus requires only $2 b$ bits to implement. Therefore, to preserve the PR property of the system, we need $b$ and $2 b$ bits to implement each multiplier in the analysis and synthesis banks, respectively.

Suppose that exact PR is not required under coefficient quantization, then we can efficiently realize the QMF bank as follows.

Implementation of $H_{k}(z)$ and $F_{k}(z)$ : At first sight, $\boldsymbol{W}_{i}$ in (41) requires 6 multipliers to implement. The total number of multipliers required to implement the analysis bank in Fig. 5 is therefore $(6 L+3)$. However, $\boldsymbol{W}_{i}$ can be factorized as

$$
\boldsymbol{W}_{i}=\left(\begin{array}{ccc}
1 & 0 & 0 \\
0 & c_{i} & 0 \\
0 & 0 & 1
\end{array}\right)\left(\begin{array}{ccc}
1 & 1 & b_{i} \\
1 & \frac{1}{a_{i} c_{i}} & 1 \\
b_{i} & 1 & 1
\end{array}\right)\left(\begin{array}{ccc}
1 & 0 & 0 \\
0 & a_{i} & 0 \\
0 & 0 & 1
\end{array}\right)
$$

Defining $\boldsymbol{W}_{i}^{\prime}$ to be

$$
\boldsymbol{W}_{i}^{\prime}=\left(\begin{array}{ccc}
1 & 1 & b_{i} \\
1 & \frac{1}{a_{i} c_{i}} & 1 \\
b_{i} & 1 & 1
\end{array}\right)
$$

$W_{i} W_{i+1}$ is implemented as follows: the two multipliers $a_{i}$ and $c_{i+1}$ can be combined, so Fig. 5 is equivalent to Fig. 9. In general, the total number of multipliers needed to implement the analysis bank (Fig. 9) is only $(4 L+3)$. The corresponding synthesis bank of Fig. 9 is shown in Fig. 10 where $\left(\boldsymbol{W}_{i}^{\prime}\right)^{-1}$ is

$$
\left(\boldsymbol{W}_{i}^{\prime}\right)^{-1}=\frac{1}{\Delta_{i}^{\prime}}\left(\begin{array}{ccc}
\frac{1}{a_{i} c_{i}}-1 & b_{i}-1 & 1-\frac{b_{i}}{a_{i} c_{i}} \\
b_{i}-1 & 1-b_{i}^{2} & b_{i}-1 \\
1-\frac{b_{i}}{a_{i} c_{i}} & b_{i}-1 & \frac{1}{a_{i} c_{i}}-1
\end{array}\right),
$$

where $\Delta_{i}^{\prime}=\left(1-b_{i}\right)\left(\left(1+b_{i}\right) / a_{i} c_{i}-2\right)$. Thus, $\left(\boldsymbol{W}_{i}^{\prime}\right)^{-1}$ appears to require 9 multipliers to implement and the overall synthesis bank of Fig. 10 requires in total $(10 L+$ 3 ) multipliers to implement. But $\left(\boldsymbol{W}_{i}^{\prime}\right)^{-1}$ can be realized as

$$
\left(\boldsymbol{W}_{i}^{\prime}\right)^{-1}=\frac{1}{\Delta_{i}^{\prime}}\left(\begin{array}{lll}
1 & 0 & 0 \\
0 & b_{i}-1 & 0 \\
0 & 0 & 1
\end{array}\right) \boldsymbol{W}_{i}^{\prime \prime}\left(\begin{array}{lll}
1 & 0 & 0 \\
0 & b_{i}-1 & 0 \\
0 & 0 & 1
\end{array}\right)
$$




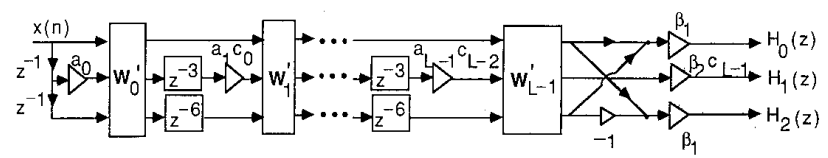

Fig. 9. Implementation of the analysis bank of the approximate PR sys-

tem.

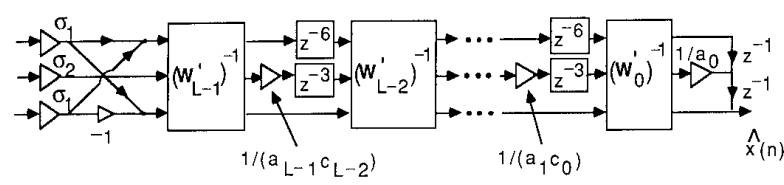

Fig. 10. The synthesis bank of Fig. 9. Here $\sigma_{1}=1 /\left(\beta_{1} \sqrt{2}\right)$ and $\sigma_{2}=$ $1 /\left(\beta_{2} c_{L-1}\right)$.

where

$$
W_{i}^{\prime \prime}=\left(\begin{array}{lll}
\frac{1}{a_{i} c_{i}}-1 & 1 & 1-\frac{b_{i}}{a_{i} c_{i}} \\
1 & \frac{1+b_{i}}{1-b_{i}} & 1 \\
1-\frac{b_{i}}{a_{i} c_{i}} & 1 & \frac{1}{a_{i} c_{i}}-1
\end{array}\right)
$$

Using the same grouping argument as in the implementation of $H_{k}(z)$, Fig. 10 is equivalent to Fig. 11 where

$$
\gamma_{1}=\frac{1}{\sqrt{2} \beta_{1}} \prod_{l=0}^{L-1} \Delta_{l} ; \quad \gamma_{2}=\frac{b_{L-1}}{c_{L-1} \beta_{2}} \prod_{l=0}^{L-1} \Delta_{l},
$$

and

$$
\eta_{i}= \begin{cases}\frac{b_{i}-1}{a_{i}} ; & i=0 \\ \frac{\left(b_{i}-1\right)\left(b_{i-1}-1\right)}{a_{i} c_{i-1}} ; & \text { otherwise. }\end{cases}
$$

The number of multipliers in this implementation is $(6 L$ +3 ). Note that all multipliers in both analysis and synthesis banks of Figs. 9 and 11 are quantized to $b$ bits and, consequently, the PR property is lost.

Implementation Complexity: To demonstrate the advantage of the lattice structure implementation in Fig. 6 over the direct-form implementation in Fig. 1, let us compare the number of multiplication and addition operations per unit time (abbreviated as MPU and APU, respectively). ${ }^{2}$

Direct-Form Implementation: A direct-form implementation of the filter triplet $\left[H_{0}(z), H_{1}(z), H_{2}(z)\right]$ would appear to require $\Sigma_{k=0}^{2} N_{k}$ MPU and $\Sigma_{k=0}^{2} N_{k}-1$ APU. Writing them in terms of $L$ and noting that $N_{0}-1=N_{2}$ $-1=12 L-5$ and $N_{1}-1=12 L-8$, it requires $\Sigma_{k=0}^{2} N_{k}=(36 L-15) \mathrm{MPU}$ and $\Sigma_{k=0}^{2} N_{k}-1=(36 L$

${ }^{2} \mathrm{~A}$ unit of time is defined to be the sampling period of the input sequence $x(n)$ in Fig. 1 .

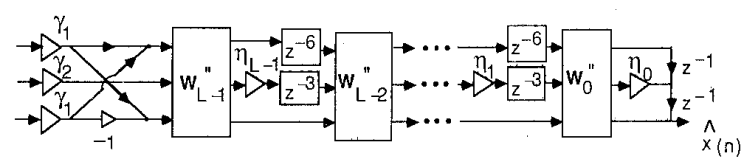

Fig. 11. Implementation of the synthesis bank of the approximate PR system.

- 18) APU. However, there are three simplifying factors involved, viz., a) the pairwise-symmetry condition; b) the linear-phase property; and c) the decimation by a factor of 3. As elaborated next, all of the above factors can be exploited to some extent.

Suppose that we implement the analysis bank of the PR system in Fig. 1 by taking advantages of only a) and b), then the linear-phase condition yields a factor of 2 saving in MPU. In addition, the MPU and APU can be further cut down by a factor of 2 since $H_{2}(z)=H_{0}(-z)$. Moreover, in the implementation of $H_{1}(z)$ which is a function of $z^{2}$, a factor of 4 saving in MPU and a factor of 2 saving in APU are obtained. In summary, the total MPU and APU required to realize the structure in Fig. 1 in direct form without utilizing the decimation factor is $(12 L-$ 4) $/ 2+(12 L-8) / 4+1=(9 L-3)=42 M P U$ and $(12 L-3)+(6 L-4)=(18 L-7)=83 A P U$, respectively.

On the other hand, let us first decimate by a factor of 3 as in Fig. 3 and implement the system at a lower rate. In doing so, we would need to realize the polyphase components $E_{k j}(z)$ directly. Since $I=1$ in this system, $\boldsymbol{E}(z)$ in (8) becomes

$$
\left\{\begin{array}{l}
\tilde{E}_{k 0}(z)=z^{m_{k}} J_{k} E_{k 1}(z), \\
\tilde{E}_{k 2}(z)=z^{m_{k}} J_{k} E_{k 2}(z),
\end{array} \quad 0 \leq k \leq 2\right.
$$

where the degree of $E_{k j}(z)$ is given in (46). Furthermore, due to the pairwise-symmetric condition, $E_{k j}(z)$ obeys

$$
\begin{cases}E_{20}(z)=E_{00}(-z), & E_{21}(z)=-E_{01}(-z), \\ E_{22}(z)=E_{02}(-z), & E_{10}(z)=\zeta_{0}\left(z^{2}\right), \\ E_{11}(z)=z^{-1} \zeta_{1}\left(z^{2}\right), & E_{12}(z)=\zeta_{2}\left(z^{2}\right),\end{cases}
$$

where $\zeta_{i}(z)$ are appropriate FIR functions. Combining (54) and (55), $\boldsymbol{E}(z)$ is

$$
\boldsymbol{E}(z)=\left(\begin{array}{lll}
E_{00}(z) & \hat{E}_{00}(z) & E_{02}(z) \\
E_{10}(z) & E_{11}(z) & E_{12}(z) \\
E_{00}(-z) & -\hat{E}_{00}(-z) & E_{02}(-z)
\end{array}\right)
$$


Due to the special form of $E_{k j}(z)$ in (55), let us consider the implementation complexity of $E_{k j}(z)$ for $k=0,2$ and for $k=1$ separately.

- $k=0,2$ : We would expect a factor of 4 in saving from the special relation of $E_{k 0}(z)$ and $E_{k 1}(z)$ for $k=0$, 2 in (56). However, the minus sign in front of $\hat{E}_{00}(-z)$ in the last row denies us a factor of 2 in saving. In other words, we cannot fully utilize the above relation. The saving is thus only a factor of 2 and, consequently, the complexity is $2(4 L-3)=(8 L-6)=34$ MPU and $4(4 L-4)+2=(16 L-14)=66$ APU. From (46) and $(55), E_{k 2}(z)$ are odd degree linear phase functions and, therefore, their complexity is $(2 L-1)=9$ MPU and $2(4 L-3)=(8 L-6)=34$ APU.

- $k=1$ : Taking advantage of the special form of $E_{1 j}(z)$ in (55), the number of MPU and APU required to realize $E_{10}(z)$ and $E_{11}(z)$ is $(2 L-1)=9 \mathrm{MPU}$ and $2(2 L$ $-2)+1=(4 L-3)=17$ APU. The remaining component $E_{12}(z)$ is an even-degree linear-phase function as well as a function of $z^{2}$, therefore, its complexity is $L=$ 5 MPU and $(2 L-2)=8$ APU. With the additional factor of 3 due to decimation, the total complexity of the analysis bank implemented in polyphase direct form is thus $(13 L-8) / 3=19 \mathrm{MPU}$ and $(30 L-22) / 3 \approx 43$ APU.

Lattice Structure Implementation: At each stage of the lattice structure in Fig. 6, the new sequences are computed at a lower rate as (Fig. 12)

$$
\left(\begin{array}{l}
v_{0}(n) \\
v_{1}(n) \\
v_{2}(n)
\end{array}\right)=\left(\begin{array}{lll}
1 & a_{i} & b_{i} \\
c_{i} & 1 & c_{i} \\
b_{i} & a_{i} & 1
\end{array}\right)\left(\begin{array}{l}
u_{0}(n) \\
u_{1}(n-2) \\
u_{2}(n-4)
\end{array}\right)
$$

The above operation requires 4 multiplications and 6 additions. Together with the multipliers $\beta_{1}, \beta_{2}$ and the 2-point DFT at the output of the lattice structure, the total complexity here is $(4 L+3)$ MPU and $(6 L+2)$ APU. Due to the decimation factor of 3 , the complexity of the analysis bank implementation using lattice structure is ( $4 L$ $+3) / 3 \approx 8 \mathrm{MPU}$ and $(6 L+2) / 3 \approx 11 \mathrm{APU}$.

Comparing the complexity of both implementations, i.e., direct form and lattice structure, we observe that lattice structure is a very efficient implementation. It should be noticed, however, that the lattice structure is not minimal in terms of number of delays because this number exceeds the filter degrees.

Comments on the Generality of the LP PR Structures: The above structures in Fig. 5 (and Fig. 6) for three-channel LP PR QMF filter bank are, by no means, general. That is, they do not cover all possible three-channel LP PR QMF banks. There are two reasons for its nongenerality. First of all, these are special cases where $I=$ 2 for the structure of Fig. 5 and $I=1$ for the structure in Fig. 6. Second, even for these special classes, the above decomposition in (26) with the choices of $\boldsymbol{A}_{i}(z)$ in (35) and $\boldsymbol{B}(z)$ in (32) does not cover all LP PR FIR analysis

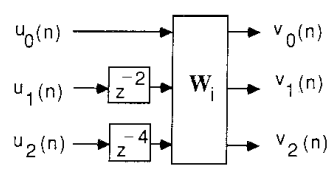

Fig. 12. One stage of the implementation of the analysis bank in Fig. 6.

banks satisfying the above constraints on filter lengths. However, the importance of these structures and the corresponding decomposition technique should not be overlooked because we are able to design filters with high attenuations which have not been done before. In other words, the filter in Example 3.1 is the first of its kind which can incorporate both linear phase and perfect reconstruction for three-channel QMF banks. Moreover, its complexity is low due to its pairwise-symmetry property. Furthermore, immediate generalization of the above structures in Figs. 5 and 6 is possible by using the same form with the appropriate dimensions. We will elaborate on this issue below.

Generalization of the Structure of $L P P R$ FIR $Q M F$ Banks for Odd M: The LP PR structure for Fig. 5 can be generalized to cover QMF banks which have more than 3 channels. Since $M$ is odd, we represent it as $M=2 L^{\prime}+$ 1. Let $H_{k}(z)$ be the analysis filters of degrees $N_{k}-1,0$ $\leq k \leq M-1$. Furthermore, assume the first $\left(L^{\prime}+1\right)$ filters to be symmetric and the remaining $L^{\prime}$ filters to be antisymmetric. $\boldsymbol{B}$ and $\boldsymbol{\Lambda}(z)$ in (32) and (35), respectively, can be generalized to be

$$
\begin{gathered}
\boldsymbol{B}=\left\{\begin{array}{ccccccccc}
1 & 0 & \cdots & 0 & 0 & 0 & \cdots & 0 & 1 \\
0 & 1 & \cdots & 0 & 0 & 0 & \cdots & 1 & 0 \\
\vdots & \vdots & \ddots & \vdots & \vdots & \vdots & \cdots & \vdots & \vdots \\
0 & 0 & \cdots & 1 & 0 & 1 & \cdots & 0 & 0 \\
0 & 0 & \cdots & 0 & 1 & 0 & \cdots & 0 & 0 \\
0 & 0 & \cdots & 1 & 0 & -1 & \cdots & 0 & 0 \\
\vdots & \vdots & \cdots & \vdots & \vdots & \vdots & \ddots & \vdots & \vdots \\
0 & 1 & \cdots & 0 & 0 & 0 & \cdots & -1 & 0 \\
1 & 0 & \cdots & 0 & 0 & 0 & \cdots & 0 & -1
\end{array}\right\}, \\
\boldsymbol{\Lambda}(z)=\left(\begin{array}{llll}
\boldsymbol{I}_{L^{\prime}} & \mathbf{0} & \mathbf{0} & \\
\mathbf{0} & z^{-1} & \mathbf{0} \\
\mathbf{0} & \mathbf{0} & z^{-2} \boldsymbol{I}_{L^{\prime}}
\end{array}\right) .
\end{gathered}
$$

The matrix $\boldsymbol{A}_{i}(z)$ satisfies the condition (33), namely, $\boldsymbol{A}_{i}(z)=\Gamma_{3} \hat{\boldsymbol{A}}_{i}(z) \Gamma_{3}$. Carrying through the same argument as in Section III, $\boldsymbol{W}_{i}$ thus takes the form

$$
\boldsymbol{W}_{i}=\left(\begin{array}{lll}
\boldsymbol{W}_{00} & \boldsymbol{W}_{01} & \boldsymbol{W}_{02} \\
\boldsymbol{W}_{10} & 1 & \boldsymbol{W}_{10} \Gamma_{3} \\
\Lambda_{L^{\prime}} \boldsymbol{W}_{02} \Gamma_{L^{\prime}} & \Gamma_{L^{\prime}} \boldsymbol{W}_{01} & \Gamma_{L^{\prime}} \boldsymbol{W}_{00} \Gamma_{L^{\prime}}
\end{array}\right)
$$




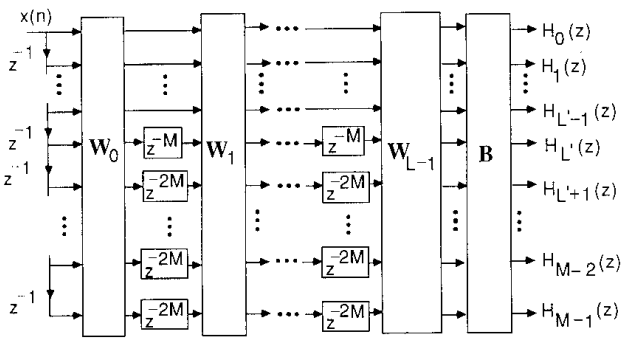

Fig. 13. The lattice structure for $M$-channel LP PR QMF analysis bank.

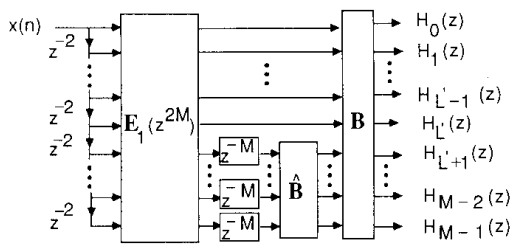

Fig. 14. The lattice structure for $M$-channel pairwise-symmetric LP PR QMF analysis bank.

where $\Gamma_{L^{\prime}}$ is defined as in (12), and $\boldsymbol{W}_{00}, \boldsymbol{W}_{02}, \boldsymbol{W}_{01}$, and $W_{10}$ have dimensions $\left(L^{\prime} \times L^{\prime}\right),\left(L^{\prime} \times L^{\prime}\right),\left(L^{\prime} \times 1\right)$, and $\left(1 \times L^{\prime}\right)$, respectively. The generalized structure is shown in Fig. 13 where $\boldsymbol{W}_{i}$ and $\boldsymbol{B}$ are as in (59) and (58), respectively.

On the other hand, the pairwise-symmetric LP PR FIR QMF analysis bank in Fig. 6 can be appropriately generalized. Let us first consider the pairwise-symmetric structure in Fig. 9 of [19]. Redrawing it using the above $\boldsymbol{B}$ in (58) yields Fig. 14. Here $\boldsymbol{E}_{1}(z)$ is as in (42) where $\boldsymbol{\Lambda}(z)$ and $\boldsymbol{W}_{i}$ are as in (58) and (59), respectively. The matrix $\hat{\boldsymbol{B}}$ in Fig. 14 is an orthogonal matrix of unit norm [19]. In summary, Fig. 14 is the analysis bank of the LP PR FIR QMF structure which yields pairwise-symmetric analysis filters.

\section{Concluding Remarks}

We have studied a subclass of the PR FIR QMF structures which yield LP analysis filters for arbitrary number of channels $M$. A number of conditions on these structures and their analysis filters are derived; subsequently, we gave a formula for the number of LP PR FIR QMF structures. To demonstrate the theory of general $M$, we elaborately studied one of the LP PR structures for the case of 3-channel. The synthesis filters in this case turn out to be linear phase with the same symmetries and orders as that of the analysis filters.

Price Paid for Perfect Reconstruction: It is often assumed that perfect-reconstruction QMF banks are much more expensive than approximate-reconstruction systems with comparable stopband attenuations for $H_{k}(z)$ 's. This impression, however, is not necessarily true. In fact, perfect-reconstruction systems implemented with lattice structures [15], [18], [19] often have computational complexity comparable to the well-known approximate-reconstruction systems.
To demonstrate this point, notice that, in Example 3.1, the computational complexity of the PR lattice is only 8 MPU and 11 APU. Suppose now that we design a linearphase equiripple FIR filter $G_{0}(z)$ with precisely the same passband and stopband ripples, and the same transition band as $H_{0}(z)$. Such a filter has order 19. Similarly, if we design a linear-phase equiripple filter $G_{1}(z)$ comparable to $H_{1}(z)$, its order is 20 . Finally, define $G_{2}(z)=G_{0}(-z)$ so that we have a linear-phase triplet $\left[G_{0}(z), G_{1}(z)\right.$, $G_{2}(z)$ ] with exactly identical properties as the perfect-reconstruction triplet $\left[H_{0}(z), H_{1}(z), H_{2}(z)\right]$. Using the above complexity calculation in the polyphase direct-form implementation, the pair of filters $G_{0}(z)$ and $G_{2}(z)$ can be realized using $13 \mathrm{MPU}$ and 28 APU. Taking advantage of the special form of $G_{1}(z)$, namely, linear phase and function of $z^{2}$, its complexity is 6 MPU and 10 APU, respectively. In total, this triplet can be implemented with $19 / 3 \approx 7$ MPU and $38 / 3 \approx 13$ APU.

In summary, the perfect-reconstruction triplet $\left[H_{0}(z)\right.$, $\left.H_{1}(z), H_{2}(z)\right]$ (implemented as a lattice) requires $8 \mathrm{MPU}$ and $11 \mathrm{APU}$, whereas the comparable nonperfect-reconstruction triplet $\left[G_{0}(z), G_{1}(z), G_{2}(z)\right]$ requires 7 MPU and 13 APU. (The non-PR triplet of course can be implemented only in direct form.) The PR system thus has competitive complexity, which appears to be counterintuitive! The fact of the matter is that the PR property permits the use of a computationally efficient lattice structure which does not exist for arbitrary (non-PR) triplets.

The principal price we actually pay for perfect reconstruction lies in the group delay created by the analysis/ synthesis system. In the PR case, this is 55 samples, whereas for non-PR triplet, this is only 20 .

\section{REFERENCES}

[1] A. Croisier, D. Esteban, and C. Galand, "Perfect channel splitting by use of interpolation/decimation/tree decomposition techniques," presented at the Int. Symp. Inform., Circuits, Syst., Patras, Greece, 1976.

[2] R. E. Crochiere and L. R. Rabiner, Multirate Digital Signal Processing. Englewood Cliffs, NJ: Prentice-Hall, 1983.

[3] V. K. Jain and R. E. Crochiere, "Quadrature mirror filter design in the time domain," IEEE Trans. Acoust., Speech, Signal Processing, vol. ASSP-32, pp. 353-361, Apr. 1984.

[4] C. R. Galand and H. J. Nussbaumer, "New quadrature mirror filter structure," IEEE Trans. Acoust., Speech, Signal Processing, vol. ASSP-32, pp. 522-531, June 1984.

[5] M. J. T. Smith and T. P. Barnwell, III, "A new filter bank theory for time-frequency representation," IEEE Trans. Acoust., Speech. Signal Processing, vol. ASSP-35, pp. 314-327, Mar. 1987.

[6] _-, "Exact reconstruction techniques for tree structured subband coders," IEEE Trans. Acoust., Speech, Signal Processing, vol. ASSP34, pp. 434-441, June 1986.

[7] J. H. Rothweiler, "Polyphase quadrature filters, a new subband cod ing technique,"' in Proc. IEEE Int. Conf. ASSP, Boston, MA, Apr. 1983, pp. 1980-1983

[8] P. L. Chu, "Quadrature mirror filter design for an arbitrary number of equal bandwidth channels,"' IEEE Trans. Acoust., Speech, Signal Processing, vol. ASSP-33, pp. 203-328, Feb. 1985.

[9] M. Vetterli, "A theory of multirate filter banks," IEEE Trans. Acoust., Speech, Signal Processing, vol. ASSP-35, pp. 356-372, Mar. 1987.

[10] - "Splitting a signal into subsampled channels allowing perfectreconstruction," in Proc. IASTED Conf. Appl. Signal Processing, Digital Filtering, Paris, France, June 1985. 
[11] K. Swaminathan and P. P. Vaidyanathan, "Theory and design of uniform DFT, parallel, quadrature mirror filter banks," IEEE Trans. Circuits Syst., vol. CAS-33, pp. 1170-1191, Dec. 1986.

[12] P. P. Vaidyanathan, "Theory and design of $M$-channel maximally decimated quadrature mirror filters with arbitrary $M$, having perfectreconstruction property, ' IEEE Trans. Acoust., Speech, Signal Processing, vol. ASSP-35, pp. 476-492, Apr. 1987.

[13] _ " "Quadrature mirror filter banks, $M$-band extensions and perfect reconstruction techniques," IEEE ASSP Mag., pp. 4-20, July 1987.

[14] M. Bellanger, G. Bonnerot, and M. Coudreuse, "Digital filtering by polyphase network: Application to sample rate alteration and filter banks," IEEE Trans. Acoust., Speech, Signal Processing, vol. ASSP24, pp. 109-114, Apr. 1976.

[15] P. P. Vaidyanathan and P. Q. Hoang, "Lattice structures for optimal design and robust implementation of two-channel perfect-reconstruction QMF banks,"' IEEE Trans. Acoust., Speech, Signal Processing, vol. 36, pp. 81-94, Jan. 1988.

[16] M. Vetterli, "Filter banks allowing perfect reconstruction," Signal Processing, vol. 10, no. 3, pp. 219-244, Apr. 1986.

[17] T. Q. Nguyen and P. P. Vaidyanathan, "Perfect reconstruction QMF structures. which yield linear phase FIR analysis filters," presented at the IEEE Int. Conf. Circuits Syst., Finland, May 1988.

[18] _ , "Two-channel perfect-reconstruction FIR QMF structures which yield linear-phase analysis and synthesis filters," IEEE Trans. Acoust., Speech, Signal Processing, vol. 37, pp. 676-690, May 1989.

[19] — - "Maximally decimated perfect-reconstruction FIR filter banks with pairwise mirror-image analysis (and synthesis) frequency responses,"' IEEE Trans. Acoust., Speech, Signal Processing, vol. 36, pp. 693-706, May 1988.
[20] J. N. Franklin, Matrix Theory. Englewood Cliffs, NJ: Prentice-Hall 1968.

[21] IMSL: A Fortran Mathematical Library.

[22] M. Vetterli and D. LeGall, "Analysis and design of perfect reconstruction filter banks satisfying symmetry constraints, "in Proc. 22nd Conf. Inform. Sci. Syst., Princeton, Mar. 1988, pp. 670-675.

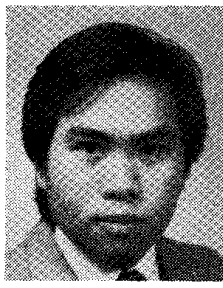

Truong Q. Nguyen (S'86-M' 89) was born in Sai gon, Vietnam, on November 2, 1962. He received the B.S. (hons.), M.S., and Ph.D. degrees in electrical engineering from California Institute of Technology, Pasadena, in 1985, 1986, and 1989. respectively.

He is currently a member of the Technical Staff at M.I.T. Lincoln Laboratory. During the academic year 1986-1987 he was a recipient of a fellowship from Aerojet General, for advanced studies. His main research interests are in digital signal processing, multirate filter bank systems, and filter design.

Dr. Nguyen is a member of Tau Beta Pi and Eta Kappa Nu.

P. P. Vaidyanathan (S'80-M'83-SM'88), for a photograph and biography, see p. 285 of the February 1990 issue of this TRANSACTIONS. 